\title{
Carcinoid tumour as a complication of ulcerative colitis
}

\author{
DAVID J. HAY \\ F.R.C.S.
}

JOHN R. N. CURT

F.R.C., M.Ch., F.R.C.S.

University of Manchester, Medical School, Hope Hospital, Salford M6 8HD

\section{Summary}

Patients with long-standing ulcerative colitis have an increased chance of developing a carcinoma of the colon, especially when the inflammatory process involves the entire colon, but no case of a carcinoid tumour of the colon occurring in a patient with ulcerative colitis has been reported.

\section{Case report}

The patient, a 54-year-old teacher, was admitted with signs and symptoms of a large bowel obstruction. He had a one-week history of central abdominal colicky pain radiating to the right iliac fossa associated with increasing constipation and abdominal distension. He had not vomited, but had been nauseated and anorexic for a few days.

Twenty years before, he had been investigated at the same hospital with sigmoidoscopy and barium enema and a diagnosis of ulcerative colitis had been made. In keeping with the accepted treatment at that time, he was started on a low residue diet. Since then, he suffered several attacks each year of blood-stained diarrhoea which were commonly precipitated by stress.

On examination he was dehydrated. The abdomen was distended with obstructive bowel sounds. The rectum was empty and sigmoidoscopy showed an injected granular mucosa which was biopsied. A diagnosis of large bowel obstruction was made which was supported by plain films of the abdomen.

In view of his dehydrated condition, intravenous fluids were given over the next $18 \mathrm{hr}$, before laparotomy. Through a right paramedian incision, the cause of the obstruction was found to be an encircling tumour of the descending/sigmoid colon. There was gross distension of small and large bowel and a perforation of the transverse colon, which was localized by omentum wrapped around the site of perforation. The large bowel itself was friable. The small bowel was decompressed with a Savage's decompressor and the hole closed with catgut and interrupted black silk sutures. A total colectomy with an ileostomy was performed, and the rectum brought out through the wound as a mucus fistula.

His progress was uneventful until the eighth postoperative day when he developed a small bowel fistula. His condition gradually deteriorated despite intensive intravenous and low residue feeding and a further laparatomy, and he died on the thirty-fourth day after his admission.

\section{Pathology}

The rectal biopsy taken at sigmoidoscopy on the day of admission showed a minimal increase in chronic inflammatory cells. There was considerable glandular loss indicative of previous damage together with oedema of the lamina propria, ap increase in plasma cells and slight polymorple infiltration indicative of a non-specific active inf flammation (Fig. 1).

Examination of the operative specimen showed the tumour to be a carcinoid tumour. It had penetrated the full thickness of the bowel wall and had infiltrated the serosal fat (Fig. 2). Elsewhere the bowel showed a variable appearance. In some areas the mucosa showed no abnormality. In others there was severe transmural acute or chronic inflammation associated with extensive ulceration and some areas where the circular and longitudinal muscle layers had been destroyed and replaced by fibrous tissue. There were no epitheloid cell granulomata.

\section{Discussion}

Patients with ulcerative colitis have an increased risk of developing carcinoma of the colon, and the incidence reported by Edwards and Truelove (1964) is between 3 and $5 \%$ of all cases. This is between 5 and 10 times greater than the risk in persons without ulcerative colitis. De Dombal et al. (1966) have shown that the risk is greatest in those with total colitis and those with distal disease have only an insignificant chance of developing carcinoma.

If the disease starts before the age of 25 years, the risk is also increased (MacDougall, 1964). In a series of 77 patients whose disease began before the age of 14 years and who subsequently developed carcinoma 


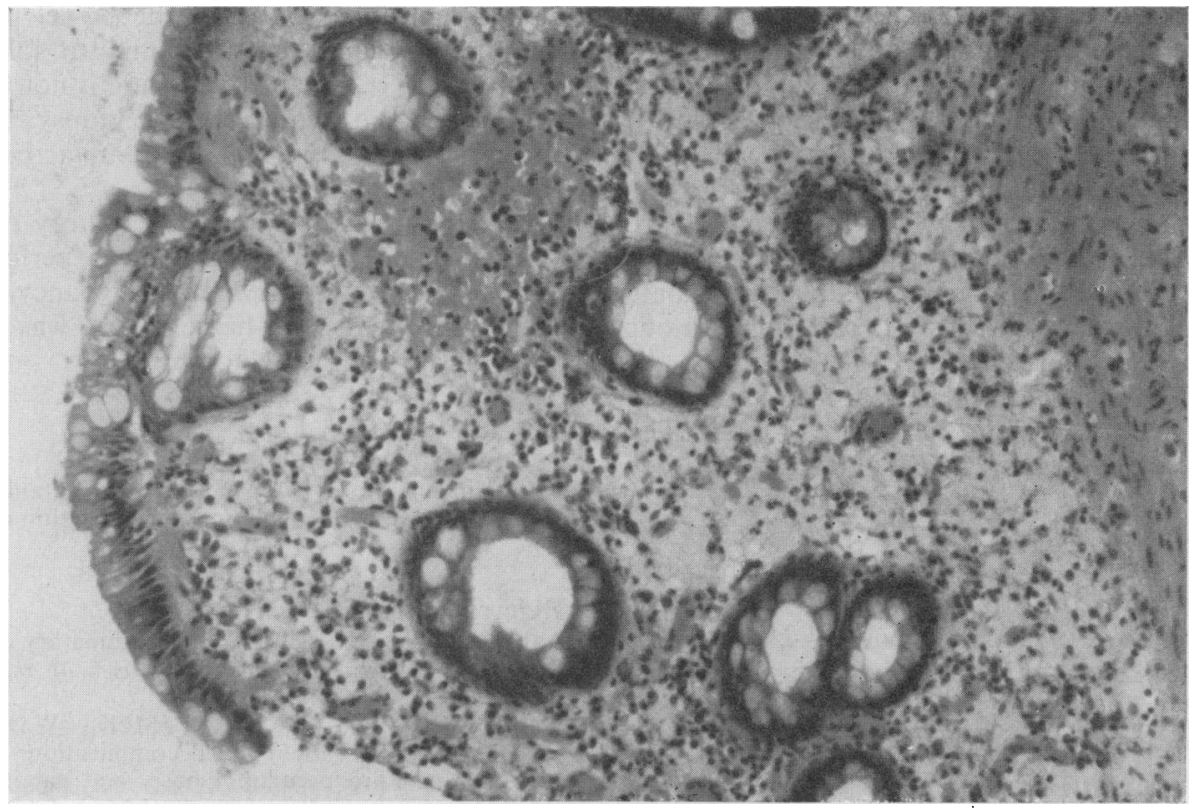

Fig. 1. Rectal biopsy HE, $\times 150$.

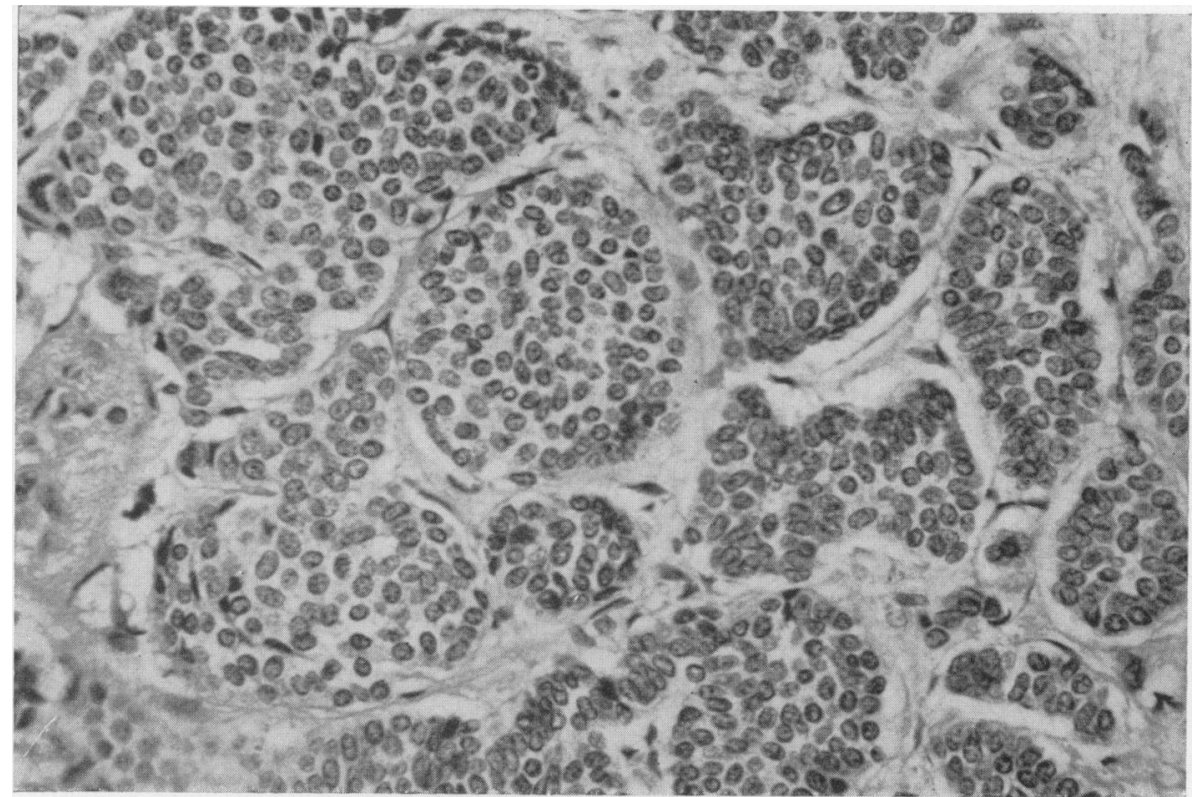

FIG. 2. Large bowel carcinoid HE, $\times 350$.

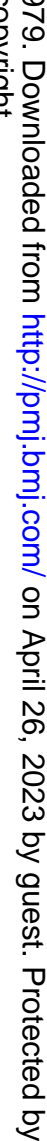


colon, Devroede et al. (1972) showed that the carcinomas were more malignant and that metastases were already present in $67 \%$ by the time of diagnosis.

Morgan's figures from St Mark's Hospital (1971) also support the fact that the tumours are often inoperable by the time they come to operation.

Carcinomas associated with ulcerative colitis are often multiple (Alexander-Williams, 1976) and poorly differentiated or mucus-secreting, but to date (1978) there has been no report of a carcinoid tumour developing in such patients.

Carcinoid tumours, or argentaffinomata, may arise anywhere that argentaffin cells are found. The tumours occur most often in the appendix, but they also occur with decreasing frequency in rectum, colon, jejunum, duodenum and stomach. The tumour is typically a small firm circumscribed nodule arising in the submucosa. In the appendix they are benign but extra-appendicular carcinoids must be considered potentially if not frankly malignant (Morgan, Marks and Hearn, 1974).

On histological examination, the cells contain cytoplasmic granules which reduce silver salts, but this is not invariable as rectal lesions do not show artentaffin granules.

Colonic carcinoid tumours usually present in the same way as the more usual adenocarcinoma, that is by large bowel obstruction or anaemia and indeed are not usually diagnosed as such until histological examination of the excised specimen has been performed. The treatment of the tumour is, on the whole, similar to that of an adenocarcinoma. In the case reported here, there is no question that the tumour was a carcinoid of the descending colon and its presentation with intestinal obstruction was not atypical.

In view of his previous investigations there is no doubt that the patient had ulcerative colitis, but at most he had only a mild form of the disease. The histological appearances of the bowel proximal to the tumour could be attributed to long-standing obstruction (Feldman, 1975) were it not for his past history and the sigmoidoscopic biopsy, the appearances of which were compatible with active ulcerative colitis.

In view of this patient's long history of recurring attacks of colitis, over a 20 -year period, he was obviously at risk to develop malignancy. It is interesting that the tumour he developed was a carcinoid and not an adenocarcinoma.

\section{Acknowledgment}

I am indebted to Dr A. W. Jones, Consultant Pathologist, Hope Hospital, for his help in the preparation of this paper.

\section{References}

Alexander-Williams, J. (1976) Inflammatory disease of the bowel: the risk of cancer. Diseases of the Colon and Rectum, 19, 579.

de Dombal, F.T., Watts, J. McK., Watkinson, G., GOLIGHER, J.C. (1966) Local complications of ulcerative colitis, stricture pseudopolyposis and carcinoma of the colon and rectum. British Medical Journal, 1, 1442.

Devroede, G.J., Dockerty, M.B., Sauer, W.G., Jackman, R.J., Strickland, G.B. (1972) Cancer of the colon in patients with ulcerative colitis since childhood. Canadian Journal of Surgery, 15, 369.

Edwards, Felicity C. \& Truelove, S.C. (1964) Course an prognosis of ulcerative colitis. Part IV. Gut, 5, 15.

Feldman, P.S. (1975) Ulcerative disease of the colon proximal to partially obstructive lesions: report of two cases and a review of the literature. Diseases of the Colon and Rectum, 18, 7, 601 .

Macdougall, I.P.M. (1964) Clinical identification of those cases of ulcerative colitis most likely to develop cancer of the bowel. Diseases of the Colon and Rectum, 7, 447.

MORGAN, C.N. (1971) Malignancy in inflammatory disease of the large intestine, Cancer, 28, 41.

Morgan, J.G., Marks, C. \& Hearn, D. (1974) Carcinoid tumours of the gastro-intestinal tract. Annals of Surgery, 180, 720.

Morson, B.C. (1966) Cancer in ulcerative colitis. Gut, 7, 425. 